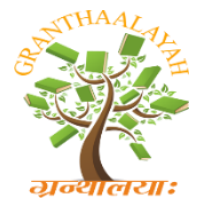

\author{
INTERNATIONAL JOURNAL OF RESEARCH \\ GRANTHAALAYAH \\ A knowledge Repository
}

Science

\title{
DIFFUSION-WEIGHTED IMAGING IN MR MAMMOGRAPHY AS AN ALTERNATIVE TO BIOPSY IN THE EVALUATION OF INTERMEDIATE NON-MASS BREAST LESIONS
}

\author{
Fatma Mohamed Awad *1 \\ ${ }^{* 1}$ MD, Radiology Department, Faculty of Medicine, Cairo University
}

\begin{abstract}
Background: Dynamic contrast-enhanced MRI is a sensitive tool for the diagnosis of breast cancer, however, its value is limited in cases of non-mass enhancement. Diffusion-weighted imaging (DWI) is promising in the diagnosis of non-mass breast lesions.

Purpose: The aim of this study is to determine the value of diffusion-weighted imaging in the evaluation of intermediate non-mass breast lesions, as an alternative to biopsy.

Materials and Methods: Thirty-three female patients between the ages of 38-56 years (mean age, 45 years) with non-mass lesions on MR mammography were included in this study. The lowest $\mathrm{ADC}$ values were obtained for the non-mass breast lesions. MR-guided core-needle biopsies were performed for 20 patients, while the other patients who refused biopsy, had yearly mammography and ultrasound every six months for two years. They also had at least one follow up MR mammography within the two years' interval.

Results: This study included 33 non-mass breast lesions detected on MR mammography. The lesion siz es ranged from 0.2 to $1.4 \mathrm{~cm}$. The morphological characteristics of the lesions and their signal intensity curves on dynamic MR Mammography were recorded. For differentiation of benign and malignant lesions, a threshold ADC value of $1.03 \times 10-3 \mathrm{~mm} 2 / \mathrm{s}$ was used. The ADC values for all the lesions ranged from $1.3 \times 10-3 \mathrm{~mm} 2 / \mathrm{s}$ to $2.6 \times 10-3 \mathrm{~mm} 2 / \mathrm{s}$.

Conclusion: Diffusion-weighted imaging is effective in the evaluation of intermediate non-mass breast lesions on MR mammography and can be used as an alternative to biopsy.
\end{abstract}

Keywords: Non-Mass Breast Lesions; DWI-MR Mammography; Apparent Diffusion Coefficient; DCIS.

Cite This Article: Fatma Mohamed Awad. (2019). "DIFFUSION-WEIGHTED IMAGING IN MR MAMMOGRAPHY AS AN ALTERNATIVE TO BIOPSY IN THE EVALUATION OF INTERMEDIATE NON-MASS BREAST LESIONS." International Journal of Research - Granthaalayah, 7(2), 171-179. https://doi.org/10.29121/granthaalayah.v7.i2.2019.1021.

\section{Introduction}

Magnetic resonance imaging (MRI) is an excellent diagnostic method in oncology (1-4). Diffusion-Weighted Imaging (DWI) measures the degree of motion of water molecules in tissues, 
which aids in the diagnosis of various diseases, including breast malignancy (5). The ADC values measured on DWI can differentiate malignant from benign breast lesions, which are different in tissue cellularity, and vascularity (6). The benefit of DW-MR mammography to characterize nonmass breast lesions is still unclear (7).

The aim of this study is to determine the value of diffusion-weighted imaging (DWI) on MR Mammography in the evaluation of intermediate non-mass breast lesions, as an alternative to biopsy.

\section{Materials and Methods}

\section{Patient Selection}

In the period from January 2015, till March 2017, 219 female patients were referred to our department for MR Mammography for further verification of sonographically-detected equivocal breast masses or for screening due to high risk of breast malignancy. From these patients, 33 patients (20 belonging to the fore mentioned former group and 13 belonging to the latter group) were included in the study, because of having non-mass lesions on MR Mammography. MRguided core-needle biopsies were performed within one month of the MRI for 20 patients, while the other patients (13 in number) who refused biopsy, had regular yearly mammography and ultrasound every six months for two years. They also had at least one follow up MR mammography within the two years' interval.

The patients' ages varied between 38 and 56 years (mean age, 45 years). Informed written consents were obtained from each patient prior to MRI and biopsy.

\section{MRI Technique}

All MRI examinations were obtained using a 1.5 Tesla MRI (Optima MR 450W, GE Healthcare, South Carolina, USA) using a bilateral phased-array breast coil. Conventional sequences of routine breast MRI were performed for all patients. The sequences used for the conventional MRI studies were axial STIR and sagittal fat-suppressed T2-weighted (TR/TE, 3850/67.4 ms and 4664/99.8 ms, respectively; slice thickness, $5 \mathrm{~mm}$; matrix, 512×512), sagittal T1-weighted (TR/TE, 542/13 ms; slice thickness, $5 \mathrm{~mm}$; matrix, $512 \times 512$ ), diffusion-weighted and contrast-enhanced threedimensional dynamic Water VIBRANT-Flex sequences (TR/TE, 7.1/3.3 ms; flip angle, $12^{\circ}$; slice thickness, $1.5 \mathrm{~mm}$; matrix, $512 \times 512$ ). One precontrast sequence was followed by six post contrast sequences for dynamic contrast-enhanced images. Gadopentetate Dimeglumine (Magnevist; Schering, Berlin, Germany) was used as a contrast medium. The contrast medium was given intravenously over $20 \mathrm{~s}$ by an automatic MR-compatible injector. The dose was $0.1 \mathrm{mmol} / \mathrm{kg}$.

The DWI sequences were performed with a two-dimensional echo-planar imaging (EPI) sequence (TR/TE, 8700/63.2 ms; slice thickness, $5.5 \mathrm{~mm}$; matrix, 256×256) in the axial plane. The images were obtained with $\mathrm{b}$ values of 0 and $800 \mathrm{~mm} 2 / \mathrm{s}$. The ADC map images were created automatically by the system.

\section{MRI Interpretation}

Contrast-enhanced dynamic sequences were first evaluated, and areas of nonmass-like enhancement were specified and correlated with the DWIs. On DWIs, the region of interest (ROI) 
was placed on the areas of nonmass-like enhancement. A standard $5 \mathrm{~mm}$ diameter circular ROI was used. Three different measurements were obtained for each area. The lowest ADC values obtained were considered the final values, rather than the mean of the three measurements.

\section{Histopathological Analysis}

Core-needle biopsies were done under MR guidance for twenty patients, using EnCor Enspire breast biopsy system, BARD, USA. Evaluation was performed using slices stained with hematoxylin-eosin.

\section{Statistical Analysis}

Statistical analysis was performed using SPSS version 20.0. McNemar's and Chi-square tests were used to compare proportions of variables. P-values $<0.05$ were considered statistically significant.

\section{Results}

This study included 33 breast lesions detected in 33 patients. Two of the patients had positive family history of breast cancer, while one of them had a past history of cancer in the other breast three years back. One of the patients had bilateral silicone breast implants.

The lesion sizes ranged from 0.2 to $1.4 \mathrm{~cm}$. The mean lesion size was $0.6 \mathrm{~cm}$. The morphological characteristics of the lesions are exhibited in Table 1.

Table 1: Morphologic characteristics of non-mass Enhancemen
\begin{tabular}{|l|l|}
\hline Pattern of Enhancement & Number of Lesions \\
\hline Distribution & \\
\hline Focal Enhancement & 23 \\
\hline Regional Enhancement & 8 \\
\hline Ductal Enhancement & 1 \\
\hline Linear Enhancement & 1 \\
\hline Internal Enhancement Pattern & \\
\hline Clustered/ Ring-like Enhancement & 3 \\
\hline Homogeneous Enhancement & 28 \\
\hline Clumped Enhancement & 2 \\
\hline
\end{tabular}

All the lesions were assigned BIRADS IVa, according to the ACR MRI lexicon. Some of the lesions had intermediate (Type II) signal intensity curves on dynamic MR Mammography, while others demonstrated benign (Type 1) signal intensity curves, as shown in Table 2

Table 2: Types of signal intensity curves of the lesions on MR Mammography

\begin{tabular}{|c|c|}
\hline Type of Curve & Number of Lesions \\
\hline Type I (Benign) & 19 \\
\hline Type II (Intermediate) & 14 \\
\hline
\end{tabular}

For differentiation of benign and malignant lesions, a threshold ADC value of $1.03 \times 10^{-3} \mathrm{~mm} 2 / \mathrm{s}$ was used. 
The lowest ADC values for all the lesions ranged from $1.3 \times 10^{-3} \mathrm{~mm} 2 / \mathrm{s}$ to $2.6 \times 10^{-3} \mathrm{~mm} 2 / \mathrm{s}$ (figures 1, 2, 3).

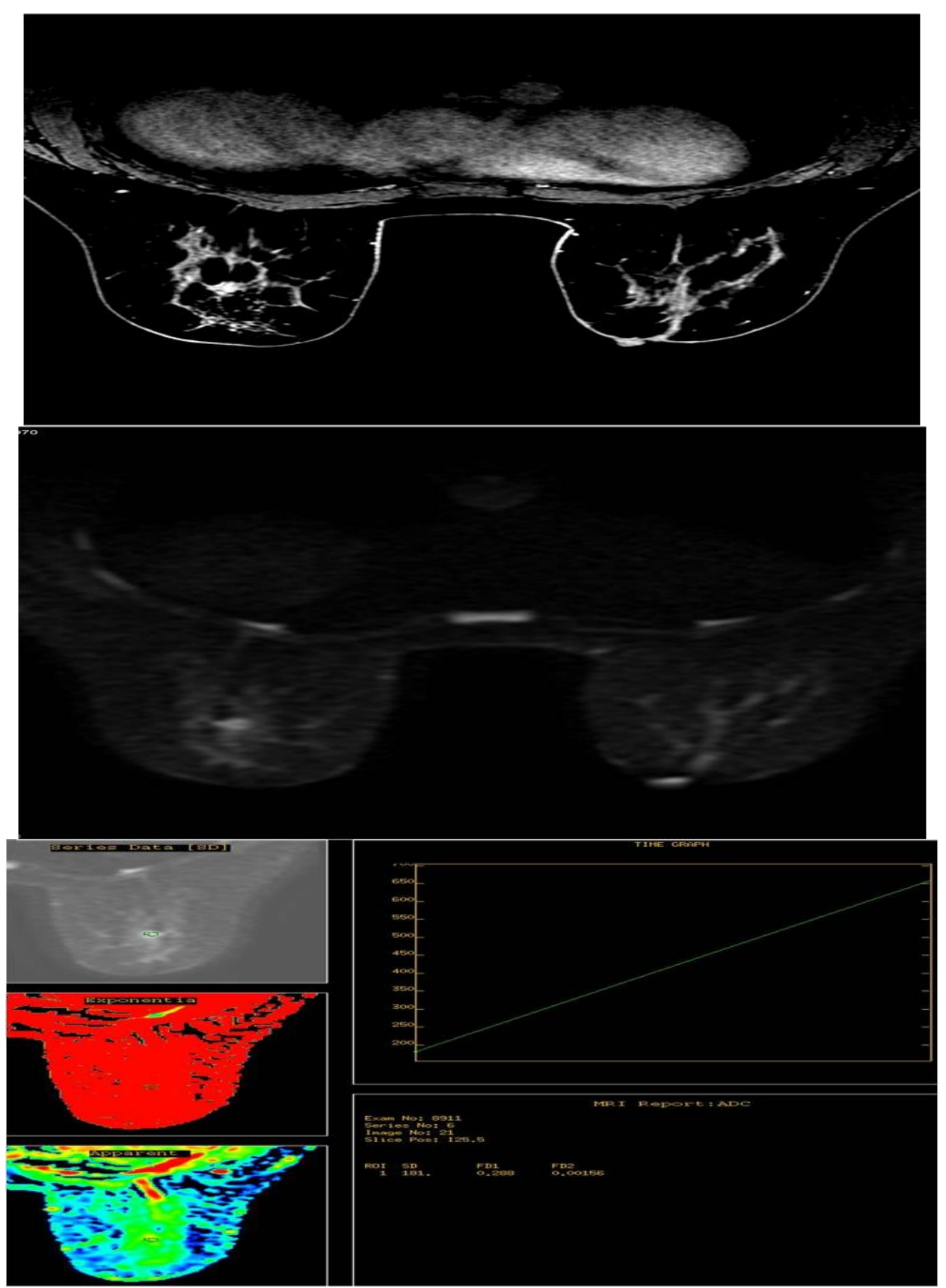

Figure 1: 46 years old woman who underwent breast MRI for further verification of right breast mass detected on mammography and ultrasound.

A, Dynamic contrast-enhanced MR image of right breast shows focal homogeneous non-mass enhancement, with type II signal intensity curve.

B, Diffusion-weighted MR image shows the non-mass lesion in the right breast.

C, Apparent diffusion coefficient (ADC) is $2.98 \times 10^{-3} \mathrm{~mm} 2 / \mathrm{s}$. MRI-guided biopsy revealed fibroadenosis. 


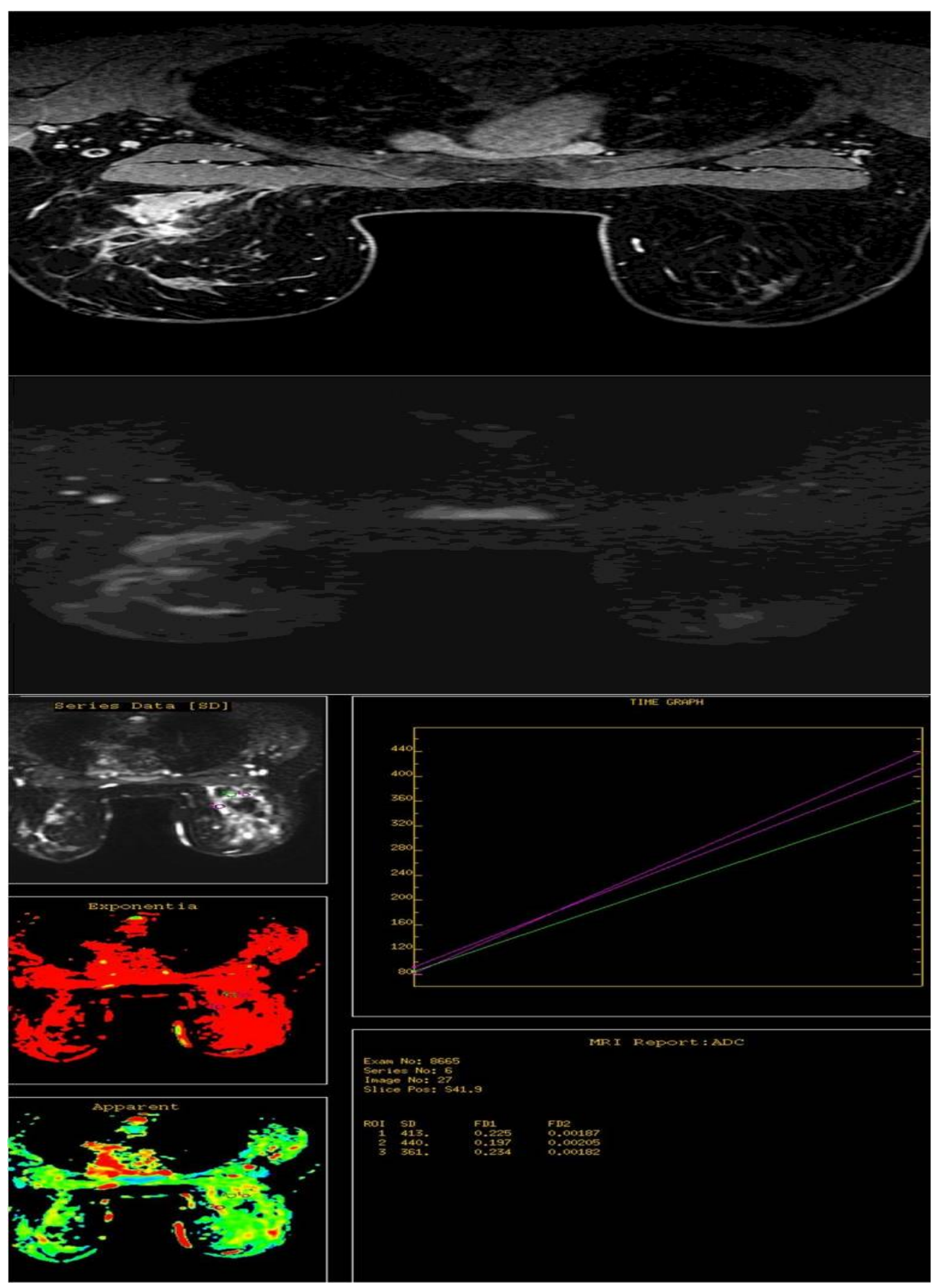

Figure 2: 39 years old woman who underwent breast MRI for suspected traumatic fat necrosis of the right breast

A, Dynamic contrast-enhanced MR image of right breast shows regional, clumped non-mass enhancement, with type I signal intensity curve.

B, Diffusion-weighted MR image shows the non-mass lesion in the right breast. C, The lowest apparent diffusion coefficient (ADC) is $1.82 \times 10^{-3} \mathrm{~mm} 2 / \mathrm{s}$. MRI-guided biopsy revealed traumatic fat necrosis. 


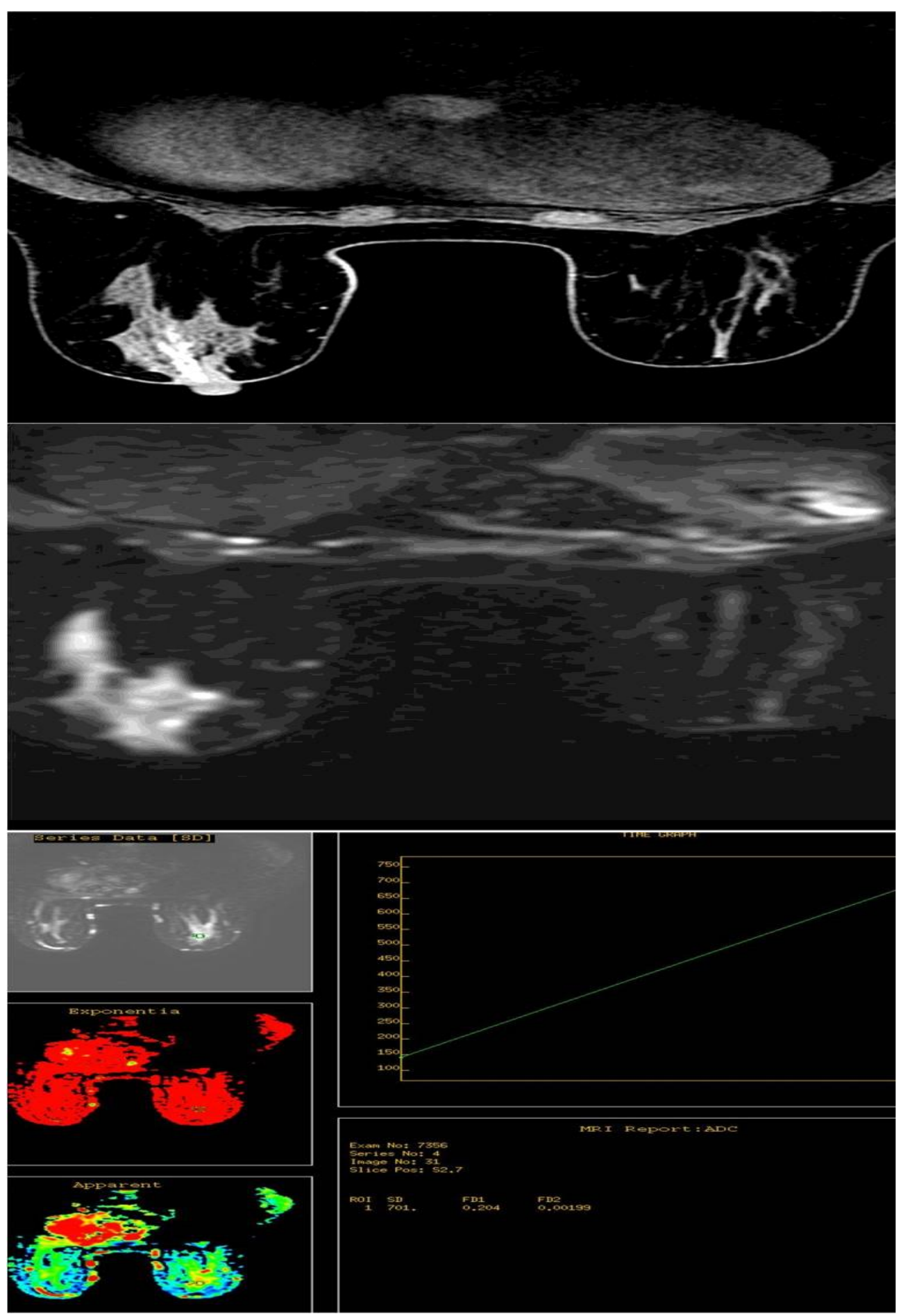

Figure 3- 45 years old woman who underwent breast MRI for further verification of right breast mass detected on mammography and ultrasound.

A, Dynamic contrast-enhanced MR image of right breast shows right retro areolar homogeneous, ductal enhancement, with type II signal intensity curve.

B, Diffusion-weighted MR image shows the retro areolar non-mass lesion. C, The lowest apparent diffusion coefficient (ADC) value is $2.04 \times 10^{-3} \mathrm{~mm} 2 / \mathrm{s}$. MRI-guided biopsy revealed fibroadenosis. 
MR-guided core-needle biopsies of twenty lesions showed all of them to be of benign etiology. The histopathological diagnoses of the lesions are shown in Table 3.

Table 3: Histopathological diagnoses of the lesions in the study

\begin{tabular}{|l|l|}
\hline Diagnosis & Number of Lesions \\
\hline Fibroadenosis & 19 \\
\hline Traumatic fat necrosis & 1 \\
\hline
\end{tabular}

\section{Discussion}

Diffusion-weighted breast imaging (DWI) has an increasingly prominent role in the radiological diagnosis of breast cancer (8).

Non-mass lesions of the breast cause high false positive results on MRI. These lesions don't exhibit the classical kinetic patterns for differentiation of benign and malignant breast lesions and thus their patterns of enhancement cannot be used for diagnosis (9). This finding is particularly significant because the most common initial manifestation of DCIS (ductal carcinoma in-situ) is non-mass enhancement (10).

This finding is in accordance with this study, because 57\% of the non-mass lesions demonstrated benign (type 1) signal intensity curves, while the rest of the lesions (43\%) demonstrated (type 2) curves. Though the difference was statistically insignificant, still a large number of benign lesions demonstrated intermediate patterns of enhancement.

Previous studies did not evaluate the role of DWI in the diagnosis of malignancy in mass lesions compared to non-mass lesions (10). In addition, no reports up to this date evaluated the sensitivity and PPV of DWI in nonmass-like breast lesions, which was the objective of this research.

In DWI, if a low b value is selected (less than $400 \mathrm{~s} / \mathrm{mm} 2$ ), the image will be affected by blood flow in the capillaries, causing pseudo- diffusion in malignant lesions $(11,12)$. DWI with higher $b$ values is more favorable, with no significant effect of the resultant signal loss on the diagnosis $(6,11,13)$. The ideal b value for DWI was $850 \mathrm{~s} / \mathrm{mm} 2(14)$. In this study, the b-value used was $800 \mathrm{~s} / \mathrm{mm} 2$, as per vendor.

Kinoshita et al (15) warned in their study that small lesions detected on MRI of the breast; less than one $\mathrm{cm}$ in diameter were easily missed. However, Partridge et al (16) stated that DWI can detect lesions of any size even the small ones. In this study, non-mass lesions of different sizes were detected on DWI, even the small ones; the size range being 0.2 to $1.4 \mathrm{~cm}$.

The final ADC value for each lesion was the minimum ADC value, because of higher sensitivity and specificity than the mean $\operatorname{ADC}$ value $(1,17,18)$.

A threshold ADC value of $1.03 \times 10^{-3} \mathrm{~mm} 2 / \mathrm{s}$ was used in this study to differentiate benign from malignant breast lesions, according to Şahin and Arıbal (19). This ADC value provided $88.5 \%$ sensitivity and $100 \%$ PPV in their study. Other studies suggested higher ADC values for differentiation between the breast lesions; however, these were the mean and not the lowest ADC 
values. In this study, the sensitivity and PPV of DWI using this threshold ADC value were also high $(100 \%)$.

All the lesions in this study were BIRADS IVa on MRI, either because of exhibiting type II signal intensity curves or because of mildly suspicious enhancement patterns, according to ACR MRI lexicon.

The limitations of this study were the small population and that no cases of ductal carcinoma in situ were included, as most of these cases were diagnosed by mammographic stereotactic biopsy of suspicious micro calcification. Further investigation is needed to verify the role of DWI in the evaluation of intermediate non-mass lesions on MR mammography.

In conclusion, diffusion-weighted imaging is effective in the evaluation of intermediate non-mass breast lesions on MR mammography and can be used as an alternative to biopsy.

\section{References}

[1] Koh DM, Collins DJ. Diffusion-weighted MRI in the body: Applications and challenges in oncology. AJR Am J Roentgenol 2007; 188:1622-1635.

[2] Englander SA, Uluğ AM, Brem R, et al. Diffusion imaging of human breast. NMR Biomed 1997; 10:348-352.

[3] Kuroki Y, Nasu K, Kuroki S, et al. Diffusion-weighted imaging of breast cancer with the sensitivity encoding technique: Analysis of the apparent diffusion coefficient value. Magn Reson Med Sci 2004; 3:79-85.

[4] Guo Y, Cai YQ, Cai ZL, et al. Differentiation of clinically benign and malignant breast lesions using diffusion-weighted imaging. J Magn Reson Imaging 2002; 16:172-178.

[5] Martincich L, Deantoni V, Bertotto I, et al. Correlations between diffusion-weighted imaging and breast cancer biomarkers. Eur Radiol 2012; 22:1519-1528.

[6] Ko ES, Han BK, Kim RB, et al. Apparent diffusion coefficient in estrogen receptor-positive invasive ductal breast carcinoma: Correlations with tumor-stroma ratio. Radiology 2014; 271:3037.

[7] Diffusion-weighted breast imaging. Clinical implementation procedure. Wenkel E, Uder m, Janka R. Der Radiologe 2014; 54:224-232.

[8] Ghai S, Muradali D, Bukhanov K, Kulkarni S. Nonenhancing breast malignancies on MRI: Sonographic and pathologic correlation. AJR Am J Roentgenol 2005; 185:481-487.

[9] Partridge SC, Mullins CD, Kurland BF, et al. Apparent Diffusion Coefficient values for discriminating benign and malignant breast MRI lesions: Effects of lesions type and size. AJR 2010; 194:1664-1673.

[10] Berg WA, Gutierrez L, NessAiver MS, et al. Diagnostic accuracy of mammography, clinical examination, US, and MR imaging in preoperative assessment of breast cancer. Radiology 2004; 233:830-849.

[11] Baltzer PAT, Benndrof M, Dietzel M, et al. False-positive findings at contrast-enhanced Breast MRI: A BIRADS Descriptor study. AJR 2010; 194:1658-1663.

[12] Woodhams R, Ramadan S, Stanwell P, et al. Diffusion-weighted imaging of the breast: principles and clinical applicaᄀtions. Radiographics 2011; 31:1059-1084.

[13] Blackledge MD, Leach MO, Collins DJ, Koh DM. Computed diffusion-weighted MR imaging may improve tumor detec $\neg$ tion. Radiology 2011; 261:573-581.

[14] Sinha S, Lucas-Quesada FA, Sinha U, et al. In vivo diffu $\neg$ sion-weighted MRI of the breast: poten $\neg$ tial for lesion characterization. J Magn Reson Imaging 2002; 15:693-704. 
[15] Bogner W, Gruber S, Pinker K, et al. Dif $\neg$ fusion-weighted MR for differentiation of breast lesions at 3.0 T: how does selection of diffusion protocols affect diagnosis? Radiology 2009; 253:341-351.

[16] Kitis O, Altay H, Calli C, et al. Minimum apparent diffu $\neg$ sion coefficients in the evaluation of brain tumors. Eur J Radiol 2005; 55:393-400.

[17] Hirano M, Satake H, Ishigaki S, et al. Diffusion-weight $\neg$ ed imaging of breast masses: comparison of diagnostic performance using various apparent diffusion coefficient parameters. AJR Am J Roentgenol 2012; 198:717-722.

[18] Şahin C, Aribal E. The role of apparent diffusion coefficient values in the differential diagnosis of breast lesions in diffusion-weighted MRI. Diagn Interv Radiol 2013; 19:457-462.

*Corresponding author.

E-mail address: ftm_wd@yahoo.co.uk 